\title{
Experiencia didáctica en la Enseñanza Secundaria. Una propuesta de ABP bilingüe para el desarrollo de las competencias clave
}

\author{
Didactic experience in Secondary Education. A bilingual PBL proposal for the devel- \\ opment of key competences
}

\author{
Diego Martínez López \\ e-mail: diegma03@ucm.es \\ Universidad Complutense de Madrid. España
}

\begin{abstract}
Resumen
Los sistemas y métodos de enseñanza tradicionales llevan ya varias décadas en la picota sin que esto haya servido para modificar los presupuestos básicos que los sustentan. A pesar de todo, son muchos los profesionales que se han decidido a dedicar sus energías a la exploración de metodologías poco comunes, como es el caso del Aprendizaje Basado en Problemas (ABP). El presente escrito, no es otra cosa que un esfuerzo por llevar este tipo de iniciativas a la enseñanza de la historia en la Educación Secundaria Obligatoria española, presentando aquí el diseño y los resultados de aplicación de un recurso ABP bilingüe en torno a la Guerra Fría, con el objetivo de demostrar no solo que otro tipo de metodologías son posibles, sino que el desarrollo de competencias clave y la conexión de la materia con el presente son imprescindibles para garantizar un aprendizaje útil y
\end{abstract} de calidad.

Palabras clave: ABP; historia; bilingüismo; educación secundaria obligatoria; metodologías docentes.

\begin{abstract}
Traditional teaching systems and methods have been pilloried for several decades without this having served to modify the basic assumptions that sustain them. Despite everything, there are many professionals who have decided to dedicate their energies to the exploration of unusual methodologies, such as the case of Problembased learning (PBL). The present paper is but an effort to take this type of initiatives to the teaching of History in the Spanish Compulsory Secondary Education, presenting here the design and the results of application of a bilingual PBL resource about the Cold War with the aim of demonstrating not only that the application of other methodologies are possible, but that the development of key competences and the connection of the subject with the present are essential to guarantee a useful and quality learning.
\end{abstract}

Keywords: PBL; history; bilingual education; mandatory secondary education; teaching methods.

Recibido / Received: 16-01-2020

Aceptado / Accepted: 26-05-2020

Publicado en linea / Online published: 01-07-2020

Cómo referenciar este artículo / How to reference this article:

Martínez López, D. (2020). Experiencia didáctica en la Enseñanza Secundaria. Una propuesta de ABP bilingüe para el desarrollo de las competencias clave. Tendencias Pedagógicas, 36, pp. 200-215. doi: 10.15366/tp2020.36.16 


\section{Introducción}

No cabe duda de que un cambio de envergadura en la enseñanza de la historia es acuciantemente necesario. Los estudios de las últimas décadas enfocados a investigar la percepción que los alumnos tienen de la materia, así como la forma de impartirla que mayoritariamente siguen los docentes así lo hacen ver $^{1}$. En este sentido, mantener los sistemas expositivos tradicionales e insistir en presentar, de forma aparentemente irremediable, los contenidos curriculares de la asignatura de forma alejada y desconectada de la realidad en la que nuestros estudiantes viven y se desenvuelven, no nos conducirá como sociedad más que a perpetuar la percepción de que la única aportación posible de la historia es la de ofrecer un compendio de datos escasamente elaborados bajo un discurso homogéneo sólo apto para las memorias más privilegiadas (Lombardi, 2000). La historia es, sin embargo, mucho más que eso. La historia es una ciencia social que, como tal, aporta un conocimiento válido que permite a los individuos comprender, situarse y actuar de forma crítica y consciente en su tiempo (Páges Blanch, 2001).

Fruto de las numerosas críticas en esta dirección que han sufrido los sistemas de enseñanza clásicos a lo largo de las últimas décadas, han surgido algunos impulsos institucionales que tratan de dar respuesta a las demandas sociales existentes en favor de una profunda renovación educativa. Aunque el resultado sigue siendo muy cuestionable, la creación del Espacio Europeo de Educación Superior, al que España se incorporó en el año 2007 a través de la aprobación de la Ley Orgánica 4/2007, de 12 de abril, por la que se modifica la Ley Orgánica 6/2001, de 21 de diciembre, de Universidades, ha permitido dar unos primeros pasos en esta dirección dentro del ámbito universitario, quedando el resto de los niveles educativos a la expectativa.

Ante esta situación, siguiendo indicaciones procedentes también del ámbito europeo, el Ministerio de Educación, Cultura y Deporte introdujo de forma reciente (2015) en el sistema educativo lo que ha sido llamado como competencias clave, una serie de conocimientos, habilidades y actitudes sociales que han de ser de ser desarrollados y adquiridos por el alumnado y que presentan como particularidad su marcada utilidad práctica para la integración inmediata del individuo en un amplio número de contextos y dinámicas sociales ${ }^{2}$. Se plantea así una teórica revolución en la enseñanza y formación de las futuras generaciones cuyo éxito se ve diariamente puesto en entredicho ante la inexistencia de una reforma estructural de amplio calado que permita el desarrollo y aplicación de este tipo de medidas. Los todavía más recientes y selectivos programas de bilingüismo no representan sino una nueva evidencia de que los sistemas institucionales clásicos deben de ser definitivamente desterrados de la educación (Enguita, 1990; Peña Calvo \& Fernández García, 2009; Obiols, 2009).

Partiendo de esta serie de premisas y con el objetivo de explorar la aplicación de nuevas metodologías de enseñanza a la Educación Secundaria Obligatoria, se presentan aquí la experiencia y resultados obtenidos a partir a de la aplicación de una propuesta educativa basada en la metodología que se conoce como Aprendizaje Basado en Problemas (ABP), la cual, en combinación con el uso intensivo de las Tecnologías de la información y la comunicación (TIC) y la incorporación de fuentes primarias, pretende evidenciar la existencia de alternativas reales y eficientes para la enseñanza de la

\footnotetext{
1 Algunos ejemplos serían: Fuentes (2002, 2003) y Suárez Suárez (2012).

2 De acuerdo con la propia Orden ECD/65/2015, de 21 de enero, por la que se describen las relaciones entre las competencias, los contenidos y los criterios de evaluación de la educación primaria, la educación secundaria obligatoria y el bachillerato, una competencia se define «como la capacidad de responder a demandas complejas y llevar a cabo tareas diversas de forma adecuada». Así, «la competencia supone una combinación de habilidades prácticas, conocimientos, motivación, valores éticos, actitudes, emociones y otros componentes sociales de comportamiento que se movilizan conjuntamente para lograr una acción eficaz». A nivel conceptual, las competencias se categorizan como un "saber hacer" que se aplica a una diversidad de contextos académicos, sociales y profesionales.»
} 
historia en las aulas de educación secundaria, todo ello sin renunciar al gran reto que supone tratar de cumplir en su totalidad los preceptos recogidos en la legislación vigente.

\section{El Aprendizaje Basado en Problemas (ABP). ¿En qué consiste?}

El Aprendizaje Basado en Problemas es un método de aprendizaje que parte de los principios de la metodología activa, el constructivismo y la formulación de problemas para establecer el núcleo de su propuesta. La metodología como tal apareció por primera vez a mediados de la década de 1950 dentro del contexto de la enseñanza de la medicina, aunque habría que esperar otra década más para que en la ciudad canadiense de Hamilton, fruto de una coyuntura económica, social y sanitaria desfavorable para la vida de las personas, el ABP comenzase a difundirse y a adquirir las características que le son atribuidas actualmente. Dicho esto, una de las mejores definiciones que se pueden encontrar de esta metodología es la dada por el propio Barrows (1986), uno de los padres del ABP, el cual lo define no como un método educativo rígido, sino como un modelo de enseñanza articulado en torno al uso de problemas como punto de arranque del aprendizaje y de la adquisición de conocimiento. Las implicaciones de la propuesta resultan claramente revolucionarias incluso hoy, pues, entre otras cuestiones, suponen como mínimo la modificación radical de los roles tradicionales asignados socialmente al binomio alumno-profesor, así como la sustitución de los canales y procesos de aprendizaje habituales (Barrón Ruiz, 1991; Savery \& Duffy, 1996; Manzanares Moya, 2010).

De forma sencilla y directa se puede decir que el ABP es una metodología de enseñanza activa que sitúa al alumno como protagonista indiscutible de su propio aprendizaje y que se articula en torno a un compendio de cuestiones y situaciones problemáticas y/o problematizadas por uno o varios profesores de materias coincidentes que se presentan en momentos determinados a un grupo de estudiantes bajo el control y supervisión de un determinado tutor. De igual modo, este método también suele definirse como un sistema curricular e instruccional que permite a los estudiantes el desarrollo simultáneo de estrategias individuales en lo que respecta a la resolución de problemas y el incremento de su nivel de conocimientos sobre una materia determinada (Vizcarro \& Juárez, 2008; Manzanares Moya, 2010).

Por otro lado, en lo que respecta a sus características concretas, lo cierto es que con su llegada a Europa a través de la Universidad de Maastricht el ABP sufrió un amplio proceso de modificación y adaptación. Así, actualmente es posible distinguir diversas modalidades de esta metodología en función del grado mayor o menor de estructuración del problema, pudiendo contraponer propuestas rígidamente confeccionadas con otras muy vagas que ceden al alumno el papel incluso de la definición del problema planteado; y el grado de intervención del profesor, yendo desde aquellos que pretenden controlar todos los canales de información hasta aquellos que adoptan un papel más puramente orientador. A pesar de ello, se pueden distinguir una serie de objetivos comunes a todas las modalidades (Barrows, 1986; Vizcarro \& Juárez, 2008; Ortiz Cárdenas, Calderón Airosa, \& Travieso Valdés, 2016):

- La estructuración del conocimiento con el objetivo de que los estudiantes sean capaces de emplearlos en cualquier otro tipo de contexto social y no sólo el académico.

- Desarrollar en los alumnos procesos eficaces de razonamiento.

- Asegurar el desarrollo de destrezas que permitan el aprendizaje autónomo.

- El empleo de problemas como vehículo principal del proceso de enseñanza-aprendizaje.

- Incrementar el nivel de motivación del alumnado a través de la generación de un interés intrínseco en resolver los problemas planteados.

- Fomentar el desarrollo de la capacidad para trabajar en pequeños grupos

- Desplazar el eje gravitacional de la enseñanza para centrar el aprendizaje en el alumno.

- Reconfigurar la labor del docente para convertirlo en un guía u orientador.

Igualmente, el método de trabajo que se suele aplicar dentro del ABP comienza con la introducción al alumnado del problema que han de resolver, lo cual obliga a los educandos a iniciar el proceso activo de análisis de la situación o cuestión planteada a fin de delimitar el problema expuesto e identificar los conocimientos ya adquiridos y que podrían ser útiles en la resolución de la tarea propuesta. Finalmente, el estudiantado debería de configurarse en pequeños grupos para tratar de resolver el problema planteado, siendo lógicamente posible tanto la búsqueda de información externa como el 
apoyo del profesor o tutor a modo de guía. Tras la resolución del problema los grupos deberían de plantear las nuevas cuestiones que han aparecido para su posterior resolución en una dinámica similar o bien proceder a asimilar los distintos conocimientos trabajados y comprendidos dentro del marco de la asignatura (Manzanares Moya, 2010).

\subsection{Problemas y tareas dentro del $A B P$}

Como ya se ha dicho, los problemas y su elaboración son una parte esencial de esta metodología, pues constituyen el principal estímulo y vehículo del aprendizaje, así como el punto de partida de este. Precisamente por ello no es una cuestión que se pueda entregar a la improvisación, sino que debe de estar preparada con antelación, siempre tratando de introducir los elementos indispensables para asegurar, por un lado, que la asignatura en que se va a emplear es perfectamente reconocible, y por el otro lado, que los objetivos perseguidos por el profesor pueden ser apreciados por el estudiantado tras un atento análisis de la propuesta realizada. Con estas premisas en mente, es habitual que los problemas adopten la forma de una narración de escasa extensión, tratando de evitar el uso de un lenguaje excesivamente técnico para su redacción y la formulación directa de preguntas (Morales Bueno \& Landa Fitzgerald, 2004; Romero Medina \& García Sevilla, 2008; Vizcarro \& Juárez, 2008).

No obstante, existen diferentes tipos de problemas. Para su clasificación podemos atender a su nivel de complejidad, su propósito curricular o a su forma. En cuanto a su complejidad, los problemas pueden ser de tres niveles, ordenados de menor a mayor complejidad de forma que el último nivel sería el correspondiente al ABP, pues serían aquellos que requieren una gran capacidad de análisis, síntesis y evaluación. Se corresponden esencialmente con situaciones reales y requieren de un proceso de investigación para su resolución, la cual no tiene porqué ser única (Morales Bueno \& Landa Fitzgerald, 2004; Romero Medina \& García Sevilla, 2008).

En lo que respecta a los problemas categorizados en función de su propósito curricular, es posible distinguir (Romero Medina \& García Sevilla, 2008):

- Problemas de orientación: Centrados esencialmente en los conceptos básicos de la materia en cuestión.

- Problemas de evaluación: Se trata de un conjunto de problemas planteados al alumnado en un contexto de examen, de forma que lo que se pretende es comprobar y evaluar los conocimientos del estudiantado a través de la resolución de las situaciones planteadas.

- Problemas de ilustración: Se trata de problemas empleados por el profesor a modo de ejemplo. Obligan a los alumnos a llevar a cabo procesos inductivos para extraer toda la información posible.

- Problemas para fomentar el razonamiento y comprensión de los contenidos: Con este tipo de problemas se persigue trabajar y desarrollar habilidades de razonamiento, análisis y síntesis.

En último lugar, se encontrarían los problemas clasificados atendiendo a su forma (Romero Medina \& García Sevilla, 2008):

- Problemas de tipo 〈pantanoso»: Caracterizados por su desorden y la presencia de numerosas cuestiones o problemas de orden secundario.

- Problemas de tipo dilema: Serían aquellos con múltiples soluciones en las que, además, habría evidentes pros y contras.

- Problemas de aplicación: Consiste en la asignación a cada estudiante de un programa de actividades que debe de hacer prosperar con éxito.

- Problemas de tipo rutinario: Serían todos los problemas típicos de cualquier libro de texto, ligados a los contenidos del tema y sin mayor trascendencia. No suelen emplearse dentro de la metodología del ABP.

\section{Metodología aplicada}

Lo primero que es necesario fijar es el bloque de contenidos elegido, el cual, en este caso concreto, se corresponde con el período histórico que comúnmente se conoce como Guerra Fría, contemplado 
dentro del currículum académico fijado para el cuarto curso de la Educación Secundaria Obligatoria (ESO) dentro del marco la asignatura de historia. Así, siguiendo el espíritu del ABP, se ha apostado por la pregunta: «How has the Cold War influenced the configuration of the world since 1945 to the present day?» («¿Cuál ha sido la influencia de la Guerra Fría en la configuración del mundo desde el año 1945 hasta hoy?») ${ }^{3}$ para definir y acotar la temática que sería trabajada en conjunción con el alumnado y en torno a la cual se llevó a cabo la confección de la unidad.

La elección del tema propuesto responde esencialmente a su idoneidad para ser trabajado bajo la metodología propia del $\mathrm{ABP}$, permitiendo la generación de constantes comparaciones y conflictos cognitivos en el alumnado; el estímulo de su pensamiento crítico autónomo; y su clara conexión e influencia en la configuración del mundo presente en el que nos hallamos insertos. En este sentido, cabe resaltar el hecho de que es precisamente esa conexión clara con la realidad actual lo que incrementa el valor de la temática seleccionada, ya que brinda una oportunidad inmejorable para elevar los niveles de interés y motivación del estudiantado a través del estímulo de su curiosidad y de sus inquietudes.

\subsection{La definición de las «reglas del juego». El contrato didáctico}

A fin de poder desarrollar satisfactoriamente los procesos de enseñanza-aprendizaje que se producirán a lo largo de las sesiones, es básico fijar las normas fundamentales que regirán tanto la relación profesor-alumno cómo las actividades propuestas, puesto que esto nos permitirá crear el marco esencial sobre el que discurrirán las distintas dinámicas en juego (Hubert, 2004). El hecho de que el ABP sea además una metodología inusual para la inmensa mayoría de los estudiantes y que su éxito dependa además del compromiso, dedicación y trabajo colaborativo fuera del aula, hace más crítica si cabe la explicitación previa de los cauces sobre los que discurrirá la actividad, pues de la comprensión y aceptación plena de los mismos por parte de los implicados en el proceso de enseñanza-aprendizaje dependerá el resultado que se obtenga al final. Así pues, las normas fijadas serían siguientes:

- El trabajo y las actividades se desarrollarán tanto de forma individual como colectiva. Será por tanto necesaria la configuración de grupos de trabajo.

- Dado el nivel, tamaño de los grupos (22 y 18 estudiantes) y que el modelo de ABP que se aplicará es el que se ha denominado como $4 x 4$ (Prieto, Barbarroja, Reyes, Motserrat, Díaz, Villarroel, \& Álvarez-Mon 2006), se han seguido las recomendaciones realizadas por Prieto, Díaz, Hernández y Lacasa (2008), fijándose así por tanto los límites de integrantes entre los valores tres y seis y el número de grupos entre cuatro y seis. Dentro de este marco, la elección y conformación de los grupos será libre, dotando por tanto de autonomía desde el comienzo a los alumnos participantes en el proceso.

- Cada grupo será responsable de la designación de un delegado y de la elección de uno de los diez subproblemas que les serán presentados durante la primera sesión. Se siguen aquí de nuevo las recomendaciones de Prieto et al. (2008) en lo referente a la conveniencia de asignación de roles previos.

- La unidad tendrá una duración mínima de siete sesiones repartidas a lo largo de tres semanas consecutivas. El tiempo de clase se reservará principalmente para las explicaciones iniciales y para la presentación de resultados.

- Las actividades por realizar recibirán un tratamiento mixto, habiendo de ser las dos primeras abordadas de manera individual y las restantes de manera íntegramente grupal.

- El seguimiento, la comunicación, la colaboración y la entrega de las tareas asignadas se realizará principalmente a través del uso de la herramienta Google Drive. Será esencial por tanto facilitar desde el primer momento una lista de correos electrónicos individuales al profesor-tutor.

- Las actividades requeridas se entregarán siempre en formato $W o r d$, atendiendo a las siguientes normas básicas:

\footnotetext{
${ }^{3}$ El recurso se puso en práctica en un aula integrada por alumnos del Programa de Bilingüismo de la Comunidad de Madrid. Ver el apartado 3.2.
} 
- Márgenes del documento: superior-inferior: $3 \mathrm{~cm}$; laterales: $2,5 \mathrm{~cm}$.

- Tipo de letra: Times New Roman.

- Tamaño de letra: 12 .

○ Interlineado: 1,5 .

- El calendario quedará fijado, en principio de forma rígida, desde el primer día, facilitando para su seguimiento un documento con las normas y fechas establecidas.

- Todos los grupos tendrán derecho a la realización de una tutoría grupal de forma previa a la realización de la exposición oral. Dicha tutoría, se celebrará en horario extraescolar y solamente se tendrá acceso a ella tras la presentación a tiempo de las actividades y tareas requeridas. Se evita así la tentación de abusar del derecho a la tutoría y se optimiza la carga de trabajo del profesor (Prieto, Díaz, Hernández, \& Lacasa, 2008).

- La última y la penúltima sesión estarán reservadas para la realización de una exposición oral y la entrega de un informe final por grupo.

- La exposición oral tendrá lugar durante la sexta sesión y tendrá como objetivo la comunicación al resto de los compañeros del aula la/s solución/es halladas por el grupo al subproblema elegido. La estructura de la presentación deberá comprender además una sucinta explicación del tema y pregunta elegida, el contexto histórico, las fuentes de información trabajadas y las líneas argumentativas principales seguidas por cada grupo. El límite máximo de tiempo por exposición se fijará entre los cinco y los siete minutos.

- La séptima sesión será dedicada a la entrega por parte de cada grupo de un informe final en el que se recojan por escrito y de forma detallada la/s respuesta/s y solución/es dadas por el grupo tanto a la cuestión particular elegida como al problema general, para lo cual se valorará positivamente la consideración de la información aportada por los demás compañeros en sus presentaciones orales. Además, se entregará al alumnado para su realización una serie de encuestas que les permitan valorar la metodología, su experiencia y el funcionamiento de su grupo de trabajo.

- La evaluación del recurso responderá al 20\% de la calificación final del trimestre.

En este punto, tras definir las reglas básicas que guiarán el recurso, conviene aclarar la fundamentación metodológica que regirá principalmente las dinámicas. En primer lugar, hay que hacer hincapié en el hecho de que este ABP responde a la filosofía del ABP 4x4 (Prieto et al. 2006), el cual recibe esta denominación principalmente porque comprende cuatro escenarios de trabajo y una división en cuatro fases para la resolución del problema planteado. Así, los escenarios en los cuales transcurrirá la actividad del alumnado se corresponderían con: el aula, en la cual se darán las pautas previas necesarias y se discutirán las respuestas finales ofrecidas por los distintos grupos a los problemas abordados; el trabajo autónomo en grupo fuera del aula y sin la presencia del tutor; la labor de investigación individual y la tutoría, la cual se mantendrá en este caso dentro del recinto, pero fuera del horario escolar.

A la vez, el procedimiento planteado consta de cuatro fases (Prieto 2006):

- Análisis: Los alumnos deberán de confrontar y analizar el problema planteado, poner en común los conocimientos de partida, tratar de identificar las lagunas de conocimiento y trazar un plan de actuación.

- Investigación: En esta fase los alumnos procederían a investigar y compartir fuertes de información de forma autónoma.

- Resolución: En este momento del proceso los estudiantes deberían de repensar el problema y emplear los nuevos conocimientos para resolverlo. Sería también la fase en la que se llevaría a cabo la elaboración de un documento y/o exposición final con la que se daría cuenta al profesor y a los compañeros de la tarea realizada y de la solución alcanzada

- Evaluación: El último paso sería llevar a cabo una evaluación de todo el proceso, incluyendo el problema y la labor del profesor.

Con la elección de este método lo que se ha buscado esencialmente es potenciar la libertad y el fomento del trabajo autónomo fuera del aula, reduciendo con ello la dependencia habitual del profesor de la que hacen gala los alumnos en los niveles medios de enseñanza. De igual modo, se pretende 
hacer hincapié en el trabajo en equipo y en el estímulo de la participación individual, cuestiones de nuevo escasamente trabajadas con los métodos tradicionales a los que los alumnos están acostumbrados. Para ello, se ha optado por reducir el número de actividades diseñadas para trabajar de forma individual sin llegar a eliminarlas, ya que se considera es imprescindible que todos los individuos partan de una serie de conocimientos básicos comunes sobre el tema a trabajar y debatir. Además, se ha considerado conveniente mantener un mínimo número de notas individuales a fin de evitar y/o paliar posibles actitudes parasitarias.

De igual forma, es conveniente resaltar el papel decisivo y particular que jugarán las TIC en el desarrollo del presente recurso. Por un lado, hay que volver a enfatizar que se utilizará la herramienta conocida como Google Drive como vehículo principal de comunicación entre los alumnos y el tutor. Se trata de una herramienta especialmente útil ya que permite suministrar y controlar el acceso de actividades y documentos, así como el trabajo colaborativo en un documento de texto conjunto sin la necesidad de concurrencia física de los participantes. Esto facilita enormemente la realización de las tareas colaborativas de forma autónoma, a la par que permite al profesor controlar el trabajo regular y continuado y realizar anotaciones que guíen la labor de los estudiantes sin necesidad de recurrir a la concertación de tutorías. Dado que el tiempo disponible para la aplicación del recurso es bastante limitado y que, en consecuencia, la preparación previa de la actividad con los alumnos deberá de ser reducida al mínimo imprescindible, esta parece ser una buena medida de control que permite al tutor reconducir la situación antes de que sea demasiado tarde.

\subsection{Adaptaciones y particularidades}

Antes de continuar, es necesario decir que el presente proyecto ha sido puesto en práctica en un centro real de educación secundaria, concretamente en dos grupos de $4^{\circ}$ de la ESO integrados en el Programa de Bilingüismo de la Comunidad de Madrid, lo cual ha requerido del empleo del inglés como lengua vehicular básica para la comunicación, así como al empleo de fuentes y recursos didácticos en dicha lengua. Fruto de esta particularidad, los ejemplos que se suministrarán a continuación aparecerán escritos en esta lengua extranjera sin traducir, a fin de mantener la mayor fidelidad posible a la actividad que se llevó a cabo. No obstante, fruto de la riqueza lingüística y de la variedad de los distintos temas tratados, ha sido posible incorporar algunos recursos y artículos en lengua castellana. Asimismo, resulta pertinente indicar que la facilidad para manejar materiales en una lengua diferente al castellano para tratar un tema como la Guerra Fría ha condicionado positivamente el diseño y confección del recurso, permitiendo la incorporación y acercamiento de fuentes primarias de información a la formación del alumnado.

\subsection{Competencias para desarrollar}

Desde comienzos de los años 2000 se realizan desde los ámbitos europeos de educación sugerencias y presiones para que todos sus Estados miembros adopten modelos educativos basados en lo que se ha denominado competencias clave. Instituciones españolas como es el propio Ministerio de Educación Cultura y Deporte definen las competencias como «un "saber hacer" que se aplica a una diversidad de contextos académicos, sociales y profesionales»". Los sistemas educativos basados en estas competencias clave lo que persiguen es que la enseñanza y el aprendizaje pasen a adoptar un carácter transversal y dinámico que contribuya al desarrollo, en principio integral, de todos los individuos insertos en dichos sistemas, facilitando con ello su integración en la sociedad en la viven y dotándolos de las habilidades y conocimientos necesarios para que estos continúen su propio desarrollo a lo largo de sus vidas.

De acuerdo por tanto a estos principios enunciados, formalmente definidos e incluidos dentro de los criterios esenciales que deben regir la evaluación de las enseñanzas primarias y medias en nuestro

4 Ver: https://www.mecd.gob.es/educacion/mc/lomce/el-curriculo/curriculo-primaria-eso-bachillerato/competenciasclave/competencias-clave.html 
país por la Orden ECD/65/2015, de 21 de enero 5 , el presente diseño didáctico ha sido elaborado manteniendo el desarrollo de las siete competencias básicas establecidas en mente, las cuales tratarán de ser estimuladas y trabajadas de la siguiente forma:

- Competencia en comunicación lingüistica: El debate de contenidos y la comunicación tanto escrita como oral de resultados son el eje básico de la metodología del ABP planteada, siendo esta, por tanto, una de las competencias que de forma directa más se trabajarán a través de la mayor parte de las actividades planteadas.

- Competencia matemática y competencias básicas en ciencia y tecnología: El acercamiento al método científico estará presente desde el comienzo de la actividad, resultando insoslayable para su desarrollo satisfactorio no solo el empleo de ordenadores e internet como herramientas de trabajo, sino la elaboración de un informe final en el que quede plasmado tanto la capacidad del estudiantado para elaborar y comunicar un discurso coherente y razonado como su soltura en el manejo de instrumentos y lenguajes técnicos aplicados.

- Competencia digital: El empleo de las TIC es indispensable para el desarrollo de la presente propuesta, pues requiere de medios informáticos tanto para la comunicación como para la consulta de materiales.

- Aprender a aprender: Puesto que el eje del aprendizaje del alumnado recae precisamente en su propia labor de investigación y trabajo autónomo, se puede considerar que este recurso les permitirá desarrollar esta competencia a través de la resolución de los problemas sugeridos y el empleo de fuentes inusuales de información, tal y como puede ser el caso de las fuentes primarias.

- Competencias sociales y cívicas: El debate constante, la tolerancia a las opiniones discordantes y la interrelación entre iguales es otro de los pilares de la dinámica del recurso. Los contenidos abordados, además, exigen el debate acerca de las distintas visiones políticas, económicas y sociales que han influido en la configuración del mundo desde la segunda mitad del siglo XX.

- Sentido de la iniciativa y espiritu emprendedor: Algunas de las actividades planteadas requieren del alumnado que demuestren su capacidad para resolver problemas, así como para trazar planes de trabajo rígidos y continuados.

- Conciencia y expresiones culturales: Dado que uno de los ejes de las dinámicas planteadas es precisamente la exploración del pasado de la civilización occidental durante el siglo XX, se requerirá del alumnado que entre en contacto tanto con la herencia cultural más inmediata del mundo actual como con estilos de vida enfrentados.

\subsection{Desarrollo de las sesiones}

\subsubsection{Sesiones introductorias}

A fin de aumentar la motivación del grupo, la confianza del mismo en la metodología del ABP y tratar de disminuir las posibilidades de desorientación y consecuente fracaso del recurso, se ha decidido dedicar dos de las siete sesiones previstas a la introducción de la metodología y las dinámicas de trabajo al alumnado.

La primera sesión, por tanto, se desarrolló en el aula durante el tiempo estipulado regularmente en el horario escolar, dotando a la misma de un formato más propio de un seminario que de una clase tradicional. El objetivo esencial no fue otro que el de realizar una pequeña introducción del origen, los fundamentos y las ventajas e inconvenientes detectados en la aplicación del ABP para acabar con una ronda de preguntas lo más amplia posible, a fin de tratar de resolver todas las dudas e inquietudes que pudiera suscitar en el estudiantado la perspectiva de tener que afrontar, de forma inmediata, la próxima unidad didáctica de la asignatura de Historia con un nuevo método de trabajo con el que no

\footnotetext{
${ }^{5} \mathrm{El}$ texto completo y la definición de las competencias puede consultarse en el siguiente enlace: https://www.boe.es/ dia-rio_boe/txt.php?id=BOE-A-2015-738
} 
estaban familiarizados. Aunque los expertos recomiendan dedicar a las tareas de entrenamiento metodológico previo un mayor número de horas y de sesiones (Prieto et al. 2006), el nivel de enseñanza en el que aplicó el recurso y las limitaciones temporales impuestas por las dinámicas institucionales obligaron a tener que reducir este tipo de actividades a su mínima expresión.

La segunda sesión fue también una actividad introductoria, pero ya más enfocada al desarrollo del recurso que a la presentación de la metodología. Para esta segunda actividad se empleó de nuevo el aula y el tiempo fijado en el horario para la asignatura, procediéndose ya a exponer las normas básicas del pacto didáctico, así como las dinámicas y ritmos de aplicación y a la recogida de una lista de correos para su inclusión en la ya citada herramienta Google Drive. Igualmente, en esta sesión se conformaron de forma libre los grupos de trabajo, se presentó al alumnado la pregunta general de este ABP: How has the Cold War influenced the configuration of the world since 1945 to the present day?, y los diez subproblemas entre los que los estudiantes debieron elegir para iniciar cuanto antes la planificación de su trabajo. Recordamos de nuevo en este punto que mantener siempre una motivación elevada es fundamental para el éxito en la aplicación del ABP, por lo que como estrategia motivacional se decidió conceder un alto grado de libertad a los estudiantes en lo que respecta tanto a la elección de sus compañeros de trabajo como del tema a estudiar.

Para la elaboración de los distintos subproblemas, los cuales se presentarán a continuación, se decidió apostar por una mezcla entre problemas de tipo pantanoso y los de tipo dilema, al considerar que ambos son las mejores tipologías de problema para desarrollar el pensamiento crítico del estudiantado, su capacidad argumentativa y la investigación autónoma, la cual, si se realiza, contribuirá enormemente a su comprensión del periodo estudiado y a conseguir una buena aproximación a la realidad de la época. Así, por tanto, los diez subproblemas diseñados fueron los siguientes:

- A bipolar world: Was it a premeditated strategy or a constructed world order?

- Germany after WWII: Was its division a preemptive tactic or a vengeance against the civil population of the country?

- Marshall Plan: An economic plan for the reconstruction of Europe or a modern Trojan horse?

- The Korean War: A showdown over a parallel or the first attempt to dominate the world?

- Spain and Europe: An ally against communism or the international legitimacy of a dictatorship?

- The Vietnam War: salvation through journalism or the failure of democracy?

- The Cuban Missile Crisis: ¿soviet provocation or part of a defensive strategy?

- The Space Race: Proof of the scientific progresses of the moment or the invention of a new weapon?

- The Non-Aligned Movement: Neutrality within the framework of a divided world or a sharp strategy for development?

- The fall of the U.S.S.R: Victory and democratic liberation or the beginning of an almost endless torture?

\subsubsection{Las actividades individuales}

A través de las distintas carpetas creadas para la ocasión en la herramienta Google Drive se hizo llegar desde el comienzo un total de tres actividades diferenciadas a cada grupo, correspondiendo estas a las dos actividades individuales que habrían de realizar, más una tercera grupal consistente en la lectura de uno o dos textos, en su mayoría periodísticos, para su posterior debate en clase.

La estructura empleada para la confección de las actividades se ha tratado de mantener en la medida de lo posible idéntica y lo más equilibrada posible, a fin de evitar un agravio comparativo entre grupos, así como una excesiva carga de trabajo para el alumnado. Manteniendo como objetivo siempre que los alumnos tuvieran la oportunidad de profundizar y conocer con un buen grado de detalle el tema escogido, incluso si no realizaban una investigación autónoma, y con el fin de dotarles de un fácil acceso a nuevas fuentes información, lo que se ha hecho ha sido plantear una primera actividad general con la que todos los estudiantes puedan introducirse y comenzar a estudiar el tema seleccionado, siempre recurriendo a fuentes online previamente filtradas y a la formulación de preguntas guía que les permitan fijar las estructuras básicas de conocimiento necesario.

Tras la realización y entrega del primer ejercicio, los alumnos realizaron una segunda actividad individual consistente en el trabajo directo con una fuente primaria o altamente especializada de información, como puede ser una tesis doctoral. El objetivo básico de este ejercicio es, como se ha mencionado en el párrafo superior, permitir a todos los individuos trabajar con un alto grado de 
detalle en el tema escogido, así como acercarles al tratamiento de fuentes de información inusuales en la educación secundaria, siempre asistidos por una serie de preguntas guía que los ayuden a «interrogan» a los documentos propuestos.

Por último, conviene ahondar algo más en la explicación de la tercera actividad, la cual como se ha dicho, si bien se realizó de forma grupal dentro del horario lectivo, requirió de una pequeña preparación previa por parte de los integrantes de cada grupo. A fin de evitar una pérdida excesiva del tiempo de clase, lo que se hizo fue facilitar a cada grupo una selección de textos actuales que demuestren cómo las dinámicas históricas estudiadas por los alumnos mantienen su relevancia y vigencia en el mundo actual. Estos textos, debieron de ser leídos de forma individual antes del día fijado para el debate, el cual se mantuvo primero entre los miembros de cada grupo para posteriormente volcar los principales puntos de discusión hacia la clase en su conjunto y enriquecer así la experiencia y la utilidad de esta.

\subsubsection{Las actividades grupales y la tutoría}

La primera actividad grupal ya ha sido descrita en el anterior subapartado por lo que no nos detendremos más en ella.

La siguiente actividad grupal de peso tuvo lugar después de la realización de la tutoría, a la cual solo tuvieron acceso aquellos grupos cuyos miembros habían cumplido con todas las tareas hasta el momento y que permitió comprobar si se estaba siguiendo el camino adecuado o, por el contrario, si era necesario realizar una intervención de urgencia. En este sentido, la tutoría resulta especialmente útil para pulsar la salud del grupo de trabajo, comprobar que todos los estudiantes participan en la actividad y que se están dando los pasos necesarios para resolver el problema planteado. Igualmente, es el momento en el que los alumnos, justo antes de realizar su exposición oral y en la antesala de tener que plasmar por escrito su informe final, pueden formular todas las dudas que les hayan surgido tanto en relación con su trabajo como en lo concerniente al método empleado. En este caso concreto se apostó por adoptar una posición intermedia entre lo que el profesor Branda (2006) ha categorizado como tutor ayudante, es decir, aquel caracterizado por su actitud servicial, y tutor desafiante, siendo este aquel que orienta su acción al desarrollo del pensamiento crítico entre sus alumnos. El objetivo perseguido es fundamentalmente el de lograr transmitir un suficiente grado de cercanía a los alumnos, lo cual es necesario para construir un agradable y productivo marco de comunicación, sin perder de vista nuestro verdadero papel como tutores.

Las dos últimas sesiones, como ya se especificó en el apartado referente al pacto didáctico, se dedicaron a la comunicación por parte del portavoz de los grupos de la respuesta dada a los distintos subproblemas, a la entrega del proyecto final por escrito y a la aplicación de los instrumentos para la autoevaluación y la coevaluación, los cuales se especificarán en el siguiente apartado.

\subsection{Criterios de evaluación}

Dado que el $\mathrm{ABP}$ es una metodología que rompe esencialmente los métodos de enseñanza-aprendizaje tradicionales, parece obvio que la forma de evaluarlo haya de ser también diferente y, en cierto modo, alejada de las prácticas habituales (Sáenz Higueras, 2009, pp. 35 y 37).

Tal y como ha quedado ya establecido, el presente recurso se ha diseñado manteniendo muy presente el desarrollo de las competencias clave definidas para el sistema educativo español, siendo por ello estas competencias o habilidades el objeto principal de evaluación. Se emplearán, por tanto, tres sistemas bien diferenciados (Fernández Sotelo y Vanga Arévalo, 2015, p. 9):

- Heteroevaluación: Se trata básicamente de la evaluación externa realizada por agentes diferentes al alumno o sus pares. En este caso concreto sería la evaluación llevada a cabo por el profesortutor. Este tipo de evaluación fue aplicada para la valoración de todas las actividades.

- Para aquellas actividades que se presentaron por escrito, se atendió a los siguientes elementos:

- Entrega a tiempo de las tareas.

- Respeto de las normas estilísticas establecidas.

- Adecuación de la respuesta ofrecida a la tarea asignada. 
- Empleo de las fuentes y herramientas facilitadas.

- Grado de comprensión de los significados.

- Correcta expresión y argumentación de las ideas.

- Capacidad para categorizar la información.

- Veracidad de los datos históricos presentados.

- Capacidad de análisis y crítica tanto de la información como de las fuentes manejadas.

- Habilidad para extraer y presentar conclusiones.

- Durante el desarrollo de la tutoría, se tuvo en cuenta principalmente:

- El grado de compleción y puntualidad en la entrega de las actividades y tareas asignadas hasta el momento.

- La capacidad de los miembros del grupo para expresarse con claridad.

- La habilidad de los individuos para relacionarse y cooperar con respeto y tolerancia entre sí.

- Grado de implicación con las dinámicas, demostrado a través del interés, investigación autónoma y la profundidad de las reflexiones ofrecidas.

- En lo que respecta a la exposición oral correspondiente a la penúltima sesión, se valoró esencialmente:

- La expresión oral.

- Control del lenguaje corporal.

- Presentación de un discurso con una estructura clara y ordenada.

- Empleo de evidencias para sustentar las ideas ofrecidas.

- Solidez de la argumentación.

- Empleo correcto de la terminología específica.

- Respeto a los tiempos marcados por exposición (5-7 minutos).

- Coevaluación: Sería aquella evaluación realizada entre iguales. Este tipo de evaluación es habitualmente empleada en el ABP ya que contribuye a aumentar el sentido de la responsabilidad de los alumnos y desarrolla su juicio crítico, ayudándolos a discernir entre el trabajo bien hecho del que no. Adicionalmente, puede llegar a crear un compromiso moral y ético profesional con sus compañeros (Bermejo \& Pedraja, 2008). En este caso específico, se ha recurrido al empleo de una encuesta anónima diseñada siguiendo la escala Likert, una escala especialmente empleada en las ciencias sociales y que permite medir realidades complejas como las opiniones (Fabila Echauri, Minami, \& Izquierdo Sandoval, 2012). En dicha encuesta, se han incluido ítems relativos a la valoración de la actividad, a la metodología y a la labor del propio tutor. Los elementos para evaluar serían por tanto los siguientes:

- El grado de satisfacción con la metodología del ABP.

- El aprendizaje realizado en comparación con los métodos habituales.

- La conveniencia de aplicar el ABP a esta y otras asignaturas.

- La valoración del trabajo colaborativo.

- La tolerancia a las opiniones de los compañeros dentro del grupo.

- Valoración dentro del grupo de trabajo de las aportaciones propias.

- Capacidad de los compañeros para aceptar opiniones críticas.

- El grado de satisfacción con el tema escogido.

- La valoración del papel realizado por el tutor.

- La conveniencia y utilidad de la tutoría.

- Autoevaluación: Se recurrió también a la evaluación por parte de los alumnos de su propio trabajo tanto a nivel individual como grupal, a fin de contribuir al desarrollo de su juicio crítico y al autorreconocimiento (Fernández Sotelo \& Vanga Arévalo, 2015). Para ello, se diseñó una nueva encuesta en la que se invitó a los alumnos a aportar información cualitativa acerca de su papel en la actividad. 
Finalmente, dado que es necesario expresar de forma cuantitativa e individual la calificación obtenida por cada alumno, la cual además equivaldría al 20\% de la calificación total del trimestre como ya se ha mencionado, únicamente queda fijar el peso que le fue concedido a cada una de las actividades. Aplicando una escala habitual de 0-10 puntos, el 40\% de la calificación correspondió a las actividades individuales, a razón de un $20 \%$ de la nota cada una, un 10\% a la actividad de debate, un $20 \%$ a la exposición oral y el 30\% restante al proyecto final. Se descartó dar un peso específico al resultado de la coevaluación a fin de asegurar la sinceridad de los participantes.

\section{Análisis de los resultados}

\subsection{Opiniones de los estudiantes}

Como ya se ha dicho, durante la última sesión programada para la realización de este ABP se pasó una encuesta anónima al alumnado a fin de que valorasen la experiencia a todos los niveles, desde su opinión de la metodología a la actuación del profesor. Como ya quedó también fijado, para el diseño de esta herramienta se ha recurrido a la escala Likert, incluyendo en este caso cuatro posibilidades de respuesta, las cuales irían a desde el «Totalmente en desacuerdo» al «Totalmente de acuerdo», pasando por las posiciones intermedias representadas por las respuestas «De acuerdo» $\mathrm{y}$ «En desacuerdo». En lo relativo a la valoración de la experiencia, se les formularon un total de 11 preguntas cerradas y 2 cualitativas en las que pudieran añadir algún tipo de comentario. Las preguntas concretas y los resultados obtenidos fueron los que siguen:

- La metodología del ABP me gusta más que el método de aprendizaje tradicional.

- La metodología del ABP me permite aprender más que con el método tradicional.

- El método del ABP me permite encontrar utilidad a lo que aprendo.

- Considero que el método del ABP facilita mi comprensión y aprendizaje de los contenidos.

- Creo que el método del ABP estimula más mi participación que el método tradicional.

- Considero que el trabajo colaborativo realizado con mis compañeros ha sido positivo para mi aprendizaje.

- Me ha gustado el método de ABP, aunque prefiero las clases tradicionales.

- El método del ABP ha incrementado mi interés por esta asignatura.

- No me gustaría que este método de enseñanza-aprendizaje se aplicase a otras asignaturas

- Considero que esta metodología me ha permitido desarrollar el pensamiento crítico

- Considero el sistema de evaluación injusto.

- ¿Cambiarías algún aspecto y/o actividad?

- ¿Qué opinión te merece el ABP?

Tabla 1.

Resultados de la encuesta anónima realizada al alumnado con relación a su valoración de la metodología y el recurso aplicado

\begin{tabular}{lllll}
\hline Pregunta & $\begin{array}{l}\text { Muy en } \\
\text { desacuerdo }\end{array}$ & En desacuerdo & $\begin{array}{l}\text { De } \\
\text { acuerdo }\end{array}$ & $\begin{array}{l}\text { Totalmente de } \\
\text { acuerdo }\end{array}$ \\
\hline 1. Gusto & 0 & 6 & 20 & 14 \\
2. Aprendizaje & 0 & 5 & 16 & 19 \\
3. Utilidad & 0 & 5 & 21 & 14 \\
4. Comprensión & 0 & 6 & 10 & 24 \\
5. Participación & 0 & 3 & 17 & 20 \\
6. Colaboración & 0 & 6 & 23 & 11 \\
7. Preferencia clase & 9 & 23 & 6 & 2 \\
$\quad$ tradicional & 0 & 15 & 20 & 5 \\
8. Interés & 11 & 20 & 6 & 3 \\
9. Aplicación otras & & & & \\
$\quad$ asignaturas & & 5 & 26 & 9 \\
10. Desarrollo del pensamiento & 0 & & 3 & 4 \\
crítico & 13 & 20 & & \\
\hline
\end{tabular}

Fuente: elaboración propia 
Observando las respuestas obtenidas se pueden extraer algunas conclusiones bastante claras y relativamente reveladoras. La primera y más obvia es que algo más del 85\% del alumnado, 34 de 40 individuos concretamente, ha manifestado su preferencia por el $\mathrm{ABP}$ antes que los métodos tradicionales de enseñanza. Igualmente, determinante es el hecho de que más de un $85 \%$ de media de los encuestados consideran que su aprendizaje, comprensión, grado de participación y utilidad de lo trabajado se ha visto positivamente afectado por esta metodología de enseñanza. La colaboración necesaria entre compañeros ha sido igualmente bien recibida, algo que puede incluso sorprender si tenemos en cuenta la orientación excesivamente individualista de los sistemas educativas actuales.

Algo paradójico resulta el hecho de que, aunque prácticamente la totalidad de la clase manifestase su gusto por la metodología del ABP, así como su mayor efectividad en comparación con las dinámicas habituales, algo más de un $20 \%$ se pronunciara en contra de abandonar los métodos clásicos. Es posible que esto se deba principalmente al miedo habitual que suscitan este tipo de experiencias nuevas en donde, además, se da un vuelco por completo no solo a la forma en la que se conciben las clases, sino a los sistemas de evaluación. El aumento de la carga de trabajo por parte del alumno es, muy seguramente, otro de los argumentos que explican esta situación. Por el contrario, es de destacar un aspecto muy positivo y es que aproximadamente un $60 \%$ de los participantes ha visto potenciado su interés por la asignatura, de lo que se puede deducir, por tanto, un lógico aumento en la motivación general de los estudiantes.

Excelentes resultan igualmente los resultados obtenidos en lo que respecta al desarrollo del pensamiento crítico, confesando más de un $85 \%$ del alumnado que, en efecto, esta actividad ha contribuido al desarrollo de su conciencia crítica, manifestándose, además, cerca de un $80 \%$ en favor de la aplicación del ABP en el marco de otras asignaturas.

En lo que respecta a las preguntas abiertas, se puede concluir que ha existido una alta satisfacción general, apuntando las quejas principalmente a la falta de tiempo y a la necesidad de insistir más en la organización y en la fundamentación previa de la metodología.

La segunda encuesta realizada fue aquella orientada a la coevaluación, autoevaluación y valoración docente. Las preguntas realizadas y resultados obtenidos fueron los que siguen:

- Mis compañeros son capaces de expresar sus opiniones de manera educada y respetuosa.

- Mis compañeros aceptan y toleran opiniones contrarias o discordantes.

- Dentro de mi grupo de trabajo me siento valorado.

- Creo que el tema planteado ha sido el correcto.

- Considero que los materiales y actividades planteadas han sido adecuadas

- Considero positivo el uso de herramientas como Google Drive

- La tutoría me ha resultado útil

- Creo que la labor del profesor-tutor ha sido adecuada:

- ¿Habrías cambiado algún aspecto relacionado con tu grupo de trabajo y/o labor del profesortutor?

- De acuerdo con mi participación y grado de implicación personalmente me calificaría con un:

- De acuerdo con la participación y grado de implicación de mis compañeros los califico con un:

Tabla 2.

Resultados de la encuesta destinada a la coevaluación, autoevaluación y valoración docente

\begin{tabular}{|c|c|c|c|c|}
\hline Pregunta & $\begin{array}{l}\text { Muy en } \\
\text { desacuerdo }\end{array}$ & En desacuerdo & $\begin{array}{l}\text { De } \\
\text { acuerdo }\end{array}$ & $\begin{array}{l}\text { Totalmente de } \\
\text { acuerdo }\end{array}$ \\
\hline 1. Expresión de opiniones & 0 & 5 & 28 & 7 \\
\hline 2. Tolerancia opiniones & 0 & 6 & 14 & 20 \\
\hline $\begin{array}{l}\text { 3. Valoración dentro del } \\
\text { grupo de trabajo }\end{array}$ & 3 & 7 & 12 & 18 \\
\hline 4. Adecuación del tema & 0 & 4 & 25 & 11 \\
\hline 5. Adecuación de materiales & 0 & 4 & 22 & 14 \\
\hline 6. Uso de las TIC & 0 & 2 & 17 & 21 \\
\hline
\end{tabular}


Fuente: elaboración propia

Como se puede apreciar fácilmente en la tabla superior, el alumnado ha transmitido una opinión muy positiva en lo que respecta tanto al trabajo en grupo realizado como al tema y materiales seleccionados, la labor del tutor y la forma en la que las TIC han sido incorporadas en el presente recurso didáctico.

Resulta reseñable el hecho de que, en general, aunque se les ha dado la oportunidad por medio de las preguntas abiertas, nadie ha hecho una crítica específica a la labor del profesor. Por el contrario, sí que se han encontrado algunos ejemplos en los que se resalta que, en ocasiones, la participación y compromiso del alumnado no es todo lo excelente que debiera ser, algo perfectamente comprensible dado el marco de enseñanza en el que nos movemos y el perfil adolescente del alumnado con el que trabajamos. De igual modo, las calificaciones otorgadas por los estudiantes a ellos mismos y a los compañeros, a pesar de no tener ningún valor de cara a la evaluación final, han sido generalmente bastante altas, en ocasiones notablemente distantes de lo percibido por el profesor, apreciándose un claro sesgo provocado por los lazos de amistad y sentido corporativo presente en la clase.

\section{Reflexiones finales}

Tras la exploración de los fundamentos de la metodología del Aprendizaje Basado en Problemas, el diseño de una unidad didáctica con esta metodología y su puesta en práctica en un aula de secundaria, podemos concluir que el ABP constituye una metodología viable y eficaz para la enseñanza de la asignatura de historia en niveles de enseñanza secundaria. Por otro lado, tras los resultados obtenidos, se concluye también que el ABP constituye una herramienta metodológica útil para la promoción y desarrollo de las competencias clave fijadas por el Ministerio de Educación y recogidas en la legislación vigente, demostrando con ello no solo la validez de los postulados constructivistas, sino su eficacia práctica para la consecución de los objetivos esenciales marcados por el actual sistema educativo. En este sentido, especialmente en lo que concierne al trabajo de las competencias clave, hay que decir que el recurso ha permitido trabajarlas todas de una forma más comprensiva y satisfactoria que con métodos tradicionales, siendo el empleo constante de tecnología, la necesidad de cooperar e interactuar de forma permanente con los compañeros y el alto grado de autonomía concedido los principales factores responsables del éxito cosechado.

Igualmente, se ha constatado el interés y predisposición favorable del alumnado ante la perspectiva de operar con dinámicas diferentes a las tradicionales a las que están más acostumbrados, siendo de especial relevancia la positividad de estimular el trabajo en grupo, la expresión oral en público y la incorporación de las TIC para aumentar la capacidad de actuación del tutor-guía, personalizar la atención y coordinar la actividad de los grupos. Esta realidad, pone de manifiesto tanto la efectividad como la conveniencia de adoptar el uso de metodologías constructivistas como el ABP para el desarrollo de la actividad educativa, ya que, como ha quedado probado, no solo tiene un gran impacto en el aumento de la motivación del alumnado, sino que además sitúa a este ante retos y situaciones poco habituales cuyo abordaje permiten el desarrollo integral de las habilidades del individuo. Quedaría pendiente la valoración a largo plazo de los efectos de estas metodologías en el aprendizaje realizado por el estudiantado, algo que no ha podido ser abordada por el momento.

No obstante, a pesar de todas las bondades registradas, se ha alcanzado también la conclusión de que el $\mathrm{ABP}$ es una metodología que demanda grandes cantidades de tiempo por parte de todos los agentes implicados. Esto supone en cierto modo, una incompatibilidad clara con los inabarcables diseños curriculares actuales y con la rigidez horaria impuesta por las instituciones escolares, pues dificultan enormemente la concesión de autonomía y organización del tiempo en el aula. Igualmente, la gran cantidad de dedicación que exige por parte del profesor la confección de este tipo de unidades o recursos imposibilita la adopción generalizada de métodos como el ABP sino se realizan previamente cambios estructurales en la organización del profesorado o se pacta la colaboración conjunta, como mínimo, entre los miembros integrantes de cada departamento.

Por otra parte, en lo que respecta al alumnado, lo novedoso de este tipo de metodologías exige la dedicación de sesiones previas para la explicación de las nuevas dinámicas de trabajo que se pretenden 
implementar, lo cual ahonda en los problemas temporales ya recogidos en el párrafo superior. $\mathrm{Al}$ mismo tiempo, la autonomía exigida al estudiantado requiere la selección de grupos formados por individuos que, en general, muestren un elevado nivel de madurez, respeto y disciplina para poder poner en práctica con un mínimo razonable de éxito propuestas basadas en metodologías constructivistas como el ABP.

Se concluye por tanto que, a pesar de las grandes bondades ofrecidas por el ABP, existen numerosas trabas estructurales que impiden su aplicación de forma generalizada en niveles de educación secundaria obligatoria. Igualmente, las características habituales del alumnado de este tipo de niveles educativos, así como cuestiones no menores relativas a las diferencias socioeconómicas y de acceso a medios digitales aún existentes, exigen prudencia y un conocimiento previo del grupo antes de tomar la decisión definitiva de poner en práctica este tipo de propuestas.

\section{Referencias}

Barrón Ruiz, Á. (1991). Aprendizaje por descubrimiento: análisis crítico y reconstrucción teórica. Salamanca: Amarú Ediciones.

Barrows, H. S. (1986). A taxonomy of problem-based learning methods. Medical education, 20(6), pp. 481-486. doi: 10.1111/j.1365-2923.1986.tb01386.x

Bermejo, F., \& Pedraja, M. J. (2008). La evaluación de competencias en el ABP y el papel del portafolio. En J. García Sevilla (Coord.) El aprendizaje basado en problemas en la enseñanza universitaria. Murcia: Universidad de Murcia.

BOE (2015). Boletín Oficial del Estado (España), núm. 25, de 29 de enero de 2015, pp. 6986-7003. Recuperado de https://www.boe.es/diario_boe/txt.php?id=BOE-A-2015-738

Fabila Echauri, A., Minami, H., \& Izquierdo, J. (2012). La escala de Likert en la evaluación docente. Acercamiento a sus características y principios metodológicos. Perspectivas docentes, 50, pp. 31-40.

Fernández Enguita, M. (1990). La cara oculta de la escuela: educación y trabajo en el capitalismo. Madrid: Siglo XXI.

Fernández Sotelo, A., \& Vanga Arévalo, María G. (2015). Proceso de autoevaluación, coevaluación y heteroevaluación para caracterizar el comportamiento estudiantil y mejorar su desempeño. Revista San Gregorio, 9(1), pp. 6-15.

Fuentes. C. (2002). La visión de la historia por los adolescentes. Revisión del estado de la cuestión en EE.UU. y el Reino Unido. Enseñanza de las Ciencias Sociales. Revista de investigación, 1, pp. 55-68.

Fuentes. C. (2003). ¿Qué visión tienen los alumnos de la historia como campo de conocimiento y como materia escolar? Íber, 36, pp. 78-88.

Hubert, M. (2004). La situación problema como facilitador de la actividad del profesor de historia. Enseñanza de las Ciencias Sociales, 3, pp. 29-38.

Lombardi, Á. (2000). La enseñanza de la historia. Consideraciones generales. Revista de Teoría y Didáctica de las Ciencias Sociales, 5, pp. 9-23.

Manzanares Moya, A. (2010). Sobre el Aprendizaje Basado en Problemas. En A. Escribano \& del Á. Valle (Eds.) El Aprendizaje Basado en Problemas (ABP): una propuesta metodológica en Educación Superior. Madrid: Narcea.

Morales Bueno, P., \& Landa Fitzgerald, V. (2004). Aprendizaje Basado en Problemas. Theoria, 13, pp. 145-157.

Obiols, G. A. (2009). Adolescencia, posmodernidad y escuela: la crisis de la enseñanza media. Buenos Aires: Kapelusz.

Ortiz Cárdenas, T., Calderón Ariosa, R. M., \& Travieso Valdés, D. (2016). La enseñanza por proyectosy el aprendizaje basado en problemas (ABP): dos enfoques para la formación universitaria desde una perspectiva innovadora. Recuperado de https://ebookcentral.proquest.com/lib/universidadcomplutenseebooks/reader.action?docID $=4508353$

Pegès Blanch, J. (2001). ¿Hacia dónde va la enseñanza de la historia y de las ciencias sociales? Apuntes para la comprensión de un debate. Éndoxa: Series filosóficas, 14, pp. 261-288. doi: 10.5944/endoxa.14.2001.5027

Peña Calvo, J.V., \& Fernández García, C. M. (Coords.) (2009). La escuela en crisis. Barcelona: Octaedro Oviedo. 
Prieto, A., Barbarroja Escudero, J., Reyes Martín, E., Montserrat Sanz, J., Díaz Martín, D., Villarroel Mareño, M., \& Álvarez-Mon Soto, M. (2006). Un nuevo modelo de aprendizaje basado en problemas, el $A B P 4 X 4$, es eficaz para desarrollar competencias profesionales valiosas en asignaturas con más de 100 alumnos. Aula Abierta, 87, pp. 171-194.

Prieto, A., Díaz, D., Hernández, M., \& Lacasa, E., (2008). Variantes metodológicas del ABP: El ABP 4x4. En J. García Sevilla (Coord.) El aprendizaje basado en problemas en la enseñanza universitaria (pp. 55-74). Murcia: Universidad de Murcia.

Romero Medina, A., \& García Sevilla, J. (2008). La elaboración de problemas ABP. En J. García Sevilla (Coord.) El aprendizaje basado en problemas en la enseñanza universitaria (pp. 37-53). Murcia: Universidad de Murcia.

Sáenz Higueras, A. (2009). ¿Cómo evaluar una actividad ABP? Revista Padres y Maestros, 323, pp. $34-$ 37.

Savery, J. R., \& Duffy, T. M. (1996). Problem Based Learning: An instructional model and its constructivist framework. En B. Wilson (Ed.) Constructivist Learning Environments: Case Studies in Instructional Design. New Jersey: Educational Technology Publications.

Suárez Suárez, M. Á. (2012). Concepciones sobre la Historia en Primaria: La epistemología como asunto clave en la formación inicial de maestros. Didáctica de la Ciencias Experimentales y Sociales, 26, pp. 73-93. doi: 10.7203/dces.26.1932

Vizcarro, C., \& Juárez, E. (2008). ¿Qué es y cómo funciona el Aprendizaje Basado en Problemas? En J. García Sevilla (Coord.) El aprendizaje basado en problemas en la enseñanza universitaria (pp. 17-36). Murcia: Universidad de Murcia. 